\title{
Transition Rule
}

National Cancer Institute

\section{Source}

National Cancer Institute. Transition Rule. NCI Thesaurus. Code C82567.

An axiom that indicates whether the subject is allowed to transition to an element of a trial, other than the next element in the previously determined sequence. 